\title{
Multitask Music-Based Therapy Optimization in Aging Neurorehability by Activation of the Informational Cognitive Centers of Consciousness
}

ISSN: 2578-0093

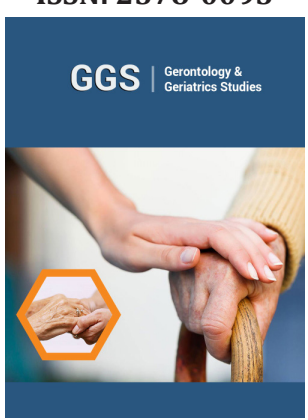

*Corresponding author: Florin Gaiseanu, $\mathrm{PhD}$, Science and Technology of Information Bucharest (Romania) and Barcelona (Spain)

Submission: 㘹 October 13, 2020

Published: 陋 November 20, 2020

Volume 6 - Issue 3

How to cite this article: Florin Gaiseanu. Multitask Music-Based Therapy Optimization in Aging Neurorehability by Activation of the Informational Cognitive Centers of Consciousness. Gerontol \& Geriatric stud. 6(3). GGS. 000639. 2020. DOI: $10.31031 /$ GGS.2020.06.000639

Copyright@ Florin Gaiseanu, This article is distributed under the terms of the Creative Commons Attribution 4.0 International License, which permits unrestricted use and redistribution provided that the original author and source are credited.

\author{
Florin Gaiseanu*
}

$\mathrm{PhD}$, Science and Technology of Information Bucharest (Romania) and Barcelona (Spain)

\begin{abstract}
The rapid increase of the old age people imposes the reconsideration of the rehabilitation techniques and procedures and/or the development of the existing ones, at least from two points of view: the limitation use of the pharmaceutical drugs because of their secondary effects in the debilitated organisms and their avoidance; the high risk of the induced anxiety states, depression or other symptoms as a consequence of the main disease, i.e. the neuro-degenerative or mobility dysfunctions, limiting again the use of such excessive drug medication. Moreover, Alzheimer's or Parknson's diseases are otherwise incurable, so the application of rehabilitating procedures like music-based therapy becomes preferential. The advances both in the identification of the specific auditory musical-sensitive circuits and of the nonmusical neuro-connections but activated indirectly by music, otherwise inaccessible by other techniques, and in the understanding of consciousness, which can be described by seven cognitive centers, allows to improve and develop the music based therapy. A multitask procedure based on the activation of these cognition centers is presented, allowing to increase the personal psychical engagement of the patient in the recommended music based therapeutic program, addressed both to the rehabilitation of the main degenerative disease and to the secondary induced dysfunctions. This procedure combines the evidence based medicine assisted by laboratory expertise for the determination of a correct diagnostic, and narrative based medicine allowing to quantify the personal engaging qualities of the patient with respect to the proposed music based program, reducing in this way the gap between these two basic procedures and bringing in a first plane the patient care, not only the disease, with beneficial consequence for the therapy efficiency and for the patient-medical care relations.
\end{abstract}

Keywords: Cognitive centers and their connections;Music-based therapy;Auditory neuropathway circuits in the brain;Ageing neurorehability;Evidence and narrative based medicine;Multitask optimization procedure

\section{Introduction}

Music is practically one of the most common and universal "language" with which the people of various realms of the earth could communicate. That is because this communication language is addressed to the human more essential and common structure, the soul [1]. Beyond of any differences of race, culture, gender, religious or education, music could be a communication "language" of feels and sentiments, so music has a profound echo in the human sensorial reactive response. For this reason, the music was used since centuries not only as a culturally specific manifestation of various human communities for intersocialization, but also as an empirical therapeutic procedure, with psychical recovery effects. In our contemporaneous informational century, when the stress of the daily activities and the pharmaceutical medication become a risk health factor especially in the old age people, the music therapy shows a powerful contributing potential with demonstrated effects in the rehabilitation of various ageing chronic diseases and neural dysfunctions like Parkinson's, Alzheimer's disease and dementia, manifested in memory impairment and executive dysfunction in daily life activities [2]. As the average life span of the actual generations is more and more increasing, the preoccupation for the application of some natural and safe treatments and techniques becomes a first objective of the medicine, activating or improving some older methodologies like meditation, or music-based therapies as a helping interventions. From this point of view, the narrative-based medicine (NBM), centered mainly 
on the care of the patient, with his particularities and distinctive personality [3] is more and more integrated as a valuable method in the evidence-based medicine (EBM) methodology, based especially on clinical analysis and expertise, rigorously applied to improve the uncertainty in diagnostic decision [4].

The large spectrum of applications of the music-based therapy (MBT) nowadays, referred to stress relaxation, recovery from anxiolytic and analgesic behavior, allowing the regulation of the heart and respiration rhythm/rate, and blood pressure in perioperative patients [5], neuro-degenerative and motor diseases, memory and learning difficulties [6], with positive results in cerebral palsy and pain in cancer survivors [7], is a recognition of the high potential of such technique to solve not only neurological health problems, but also allowing to study and understand the activity of various zones of the brain, otherwise non-accessible with other non-destructive and non-invasive methods [8]. However, the personalization healthcare supposes an adequate opening of the communication channels with the patient, the knowing and understanding of the patient's particularities with respect to the rehabilitation objective and method. Therefore, the aim of this paper is to cover the lack of a systematic (NBM) methodology based on a specific communication and quantitative evaluation of the particularities of the patient, specifically in multitask treatment of ageing diseases and their derivative complications, on the basis of the application of a correct, coherent, systematic and full description of the cognitive architecture of the human consciousness.

\section{The Music Neuro-Connections from the Perspective of the Informational Functions of the Brain}

Recent studies defined with higher accuracy the auditory-limbic pathway connections with the sound and the intimate mechanisms and pathways of the music-induced emotive states in the brain $[9,10]$, as it is shown schematically in the right side of (Figure 1). In this figure, the region of the auditory limbic system (drawn in white color), is situated in the ear/cochlear zone of the brain, connected with the local nervous nuclei which create the auditory sensation and with the central nervous nuclei of amygdala and brainstem, which give rise a somato-motor and automatic response of the nervous system to the sound signals.

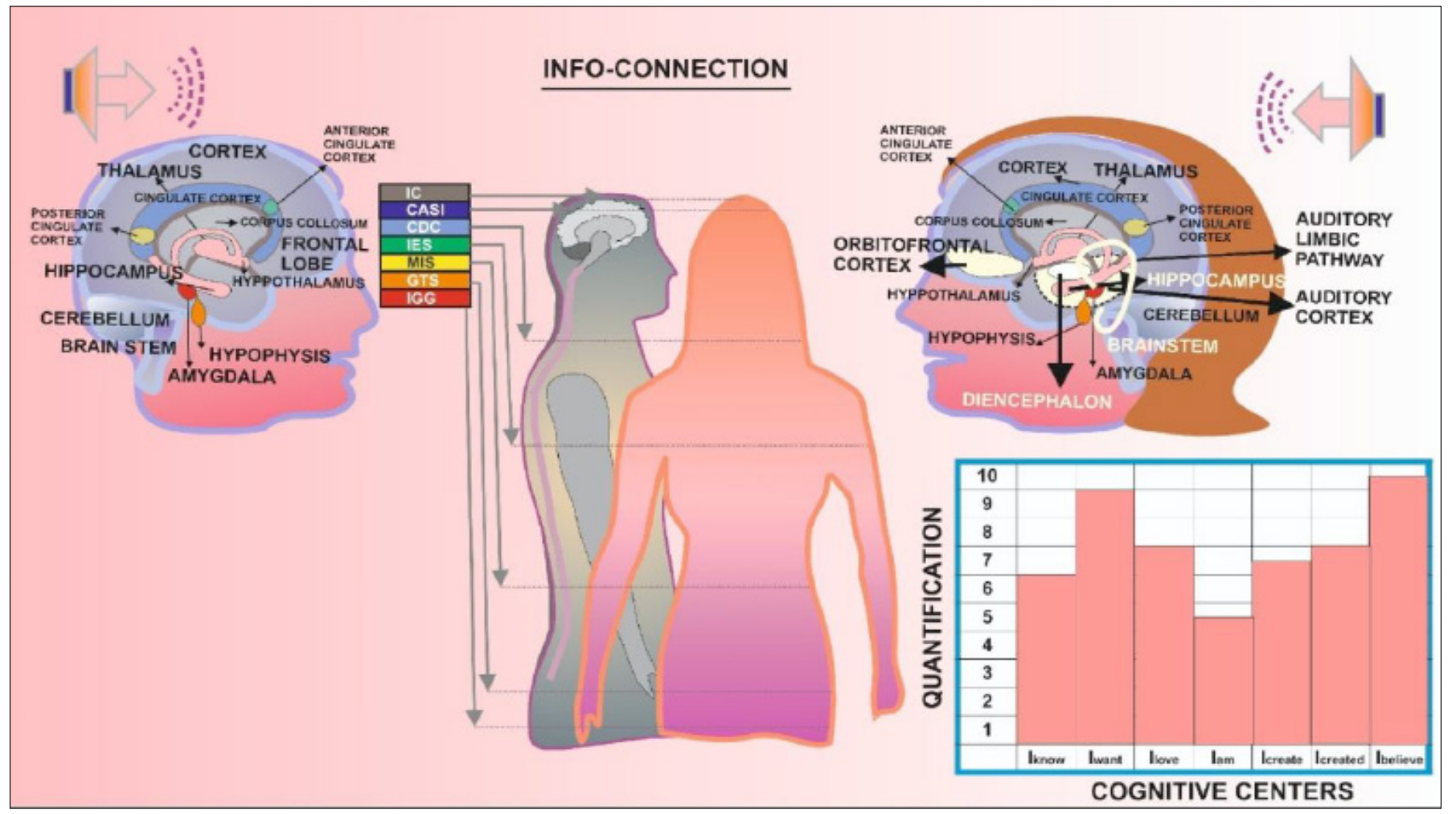

Figure 1: Schematic representation of the informational circuits in human organism (left side), their connections with the brain and with the body regions (indicated by black arrows), according to the informational structure of consciousness, and the specific neuro-pathway circuits, shown in the upper right side of the figure. In the bottom right side is inserted an appraisal diagram, showing the activation level of the cognitive centers with respect to the proposed objective, i.e. the music-based therapy program.

By using some modern tools of investigation based on imaging techniques, there were detected according to these studies four main regions of the brain involved in the transmission and processing of the audio signals [10]: the orbitofrontal cortexassimilated with subconsciousness, hippocampus, stimulating the happiness/satisfaction, brainstem, leading the vitalization/ reinforcement, and diencephalon, which is a subcortical region between cortex and brainstem, referring especially to thalamus/ subthalamus, hypothalamus and pineal gland (hypophysis), managing the pain and pleasure sensations. This region is marked in Figure 1 by a white area. We have to observe therefore that besides the specialized hearing nerve and auditory cavities, various 
other neuro-connections and brain areas like the auditory cortex, projecting to amygdala, orbitofrontal cortex and the cingulate cortex transitory region, represented also in the right side of Figure 1, participate to the sound processing. The basal ganglia nuclei, involved in the motor actions and learning, are the inferior active region of diencephalon, involved also in the music processing. The brainstem, as an inferior zone of the brain, receives the sound information transferring it to the body, in which mecanoreceptors sensitive to the sound vibrations are distributed everywhere. From this description we have to observe the large spectrum of functions influenced by the sound signals, from perception to emotional and motor-assisted response.

Two main aspects are to be pointed out from these studies: (1) the implication of amygdala, anterior cingulate cortex, the orbitofrontal cortex, somatosensory cortex and thalamus in reward pleasurable properties of the brain, explaining the reduction of the pain as effect of the music [10-12]; (2) hippocampus was found to participate in attachment relations, activating joy, happiness or sadness [10]. Analyzing from the informational point of view the music perception/reactive response by the brain, we have to note that music is actually information, so the analysis of how the organism responds to this information signifies actually to understand the consciousness structure and mechanisms and how consciousness is coupled with the external reality $[1,13]$. However, the interpretation of the same informational signal is a discussable question, because every person disposes of own distinct characteristics to interpret information, both because these characteristics themselves are distinct, and the personal experience [14] and decision criteria are also different [15]. On the other hand, we have to observe that music is not a simple signal, it is a multi-frequency polyphonic and rhythmic complex of sounds, their vibrations inducing emotional states in the human organism, either good or bad, but any way impressive. More variables are to be introduced in the therapy problem when the aging process is taken into account, because of various degradation phenomena associated to the nervous system should be considered, as will be detailed in the subsequent section.

The interaction of information with the human organism starts with the perception process of information by sensors. This information is transmitted by neuro-connections to the brain, which stores it for short and longer time in memory, so a Center of Acquisition and Storing of Information (CASI) could be defined as a sum of the involved brain areas (Fig. 1 left side), which are: the prefrontal cortex (short-term memory), hippocampus (long-term memory) and cerebellum, when the repetitive stereotype chains of actions are acquired as skills [16]. The primary reactive response to received information is perceived as emotions or sensations, which could be automatically triggered in the informational circuit of the brain including amygdala [17], or felt as positive or negative emotional states, depending also on the personal acquired experience and judgment criteria. Therefore, an Info-Emotional System (IES) could be defined, supported mainly by the limbic system of the brain, which includes amygdala, hippocampus, hypothalamus and cingulate gyrus. The conscious analysis and responsive decision, either related to an internal or external stimulus or signal, is given in the brain areas represented especially by the two cortical hemispheres, supporting the activity of the Center of Decision and Command (CDC). As we have to observe, a part of the IES reactive response is automatic, and another part is consciously detected and contributes to the decision-making process. As in the decisional processes mainly intervenes only the conscious emotional information, an Operative Informational System (OIS) could be defined as OIS=CASI+CEC+IES, which operates for rapid or medium-term adaptation to the environmental/internal conditions. The attitude is therefore an informational output response to an input information, which can be manifested through an execution commands to the motor elements of the body or maintained in memory as a reference experience for further utilization.

The programmed informational system (PIM) maintains the correct functioning of the organism from the following point of views: body maintenance by the metabolic processes, defined as the maintenance information system (MIS), connected especially to brainstem, medulla and the spinal cord (digestion, breathing, heart rate, body temperature, blood pressure and distribution, chronological processes) [18], genetic transmission system (GTS), and info-genetic generator of (inherited) information (IGG), connected especially to hypophysis and hypothalamus [17], so that: PIS=MIS+GTS+IGG. An additional center of PIS can be defined as Info-Connection (IC), which determines the right orientation of information according to the survival criteria, intervening also in phenomena like near-death experiences (NDEs), religious and mystic experiences (RMEs) [16] and extra sensorial processes $[19,20]$, connected especially to cingulate cortex $[3,21]$.

The info-related systems are projected in consciousness according to the informational model of consciousness (IMC) [16] as following: CASI=>Iknow (memory [14]), CDC=>Iwant (decision [15]), IES=>Ilove (emotions [22]), MIS=>Iam (personal state, vitality), GTS $=>$ Icreate (creative impulses and skills), IGG $=>$ Icreated (inherited predispositions, inherited skills [23]), IC $=>$ Ibelieve (trust and confidence), which are actually the cognitive centers of consciousness, fully describing the own personality. The personification expressed by "I" highlights that actually each person is the holder of his/her info-experience, either inherited and accumulated during the life, as a result of own mode to perceive or interpret the external and internal reality [1]. If we refer to the MBT, we have to observe therefore that this procedure should be adjusted to particular perception and interpretation characteristics of each patient, for efficient rehabilitation effects.

\section{Multitask Optimization of the Music Based Therapy}

In terms of information, the specific sound circuits marked in Figure 1 right side (white regions) are related with the following cognitive centers of consciousness: orbitofrontal cortex is related with Iknow (short-time memory), hippocampus with Iknow (long-time memory) and Ilove (emotional memory), diencephalon (white letters) with Ilove (connected mainly to the limbic system) and brainstem with Iam ("vitality" and equilibrium/health status [3]), with projections in Ibelieve [21,24], Iwant (decision) and 
Iam (motor skill system acquirement). This shows the remarkable influential power could have the info-music agents on the entire organism and its wellbeing, if an MBT program is suitably applied according to the particular characteristics of the patient. In the old age adults, there is a high risk for degenerative neurologic dysfunctions and diseases, specifically Alzheimer's with dementia manifestation and followed by the Parkinson's disease (otherwise not curable diseases), doubled by secondary effects like anxiety, depression and memory dysfunctions states. Mobility dysfunctions are also frequent problems with which the old age adults are confronted, giving rise to subsequent secondary effects like anxiety, depression or pain, so multitask MBT should be therefore applied, including a specific program for each of them, but performed in a single common cycle. MBT is beneficial from this point of view for neurorehabilitation in all these various aspects, from mobility to pain treatment, taking into account that the auditory areas are connected with motor functions (execution elements managed by Iwant), emotions (Ilove), motivation (Ibelieve), memory (Iknow) and with cognition in general, so rhythm and music are powerful means to stimulate non-auditory regions of the brain, both affected by neurologic and psychiatric disorders $[2,25]$. The beneficial application of a particular MBT fitted program on personal cognitive characteristics was recently demonstrated, showing improvements in the rehabilitation by means of MBT, if music is chosen by the participants themselves [25].

All these data indicate that a particular application of MBT as a function not only on the medical diagnostic established by EBM procedures, but also on the specific psychical characteristics of the patients should be carefully prepared. On the basis of the new results concerning the informational systems of the human body and the corresponding associated cognitive centers as presented above, a systematic suitable procedure to activate these cognitive centers for a better coupling with the rehabilitation music-based program of each patient could be prepared and applied as following: (1) the diagnostic determination (by EBM procedures) of the main disease and of the secondary derivatives dysfunctions, and the treatment prescription; (2) the application of a NBM interviewtype test over each of the seven cognitive centers referred to the accommodation/concordance of the recommended music with own experience/expectative, to detect the specific capabilities/ qualities of the patient to integrate in the prescribed MBT, and the quantification of these qualities on a reference diagram (Fig. 1 bottom right side); (3) the application of the recommended MBT program; (4) the application again of a NBM-type test to observe the feedback response, for further corrections of the music applied program.

The NBM type test should explore each cognitive center as following: Iknow - to observe the previous experience concerning the proposed music category; Iwant - determining the level of the personal decision involved in a specific MBT; Ilove - referring to the emotional/attachment inspired by a specific music category; Iam the "vitalization" effect of the proposed music and own self-control capacity; Icreate - the creative participation to a specific proposed MBT; Icreated - the concordance with own predispositions and abilities; Ibelieve - the trust and confidence in the proposed music program. Each specific cognitive quality associated to each center should be quantified on a scale of 10 units, as presented in the diagram of the right bottom side of Figure 1, taking into account for instance the following associated qualities, denoted by the symbol Q: earlier experience - Q(Iknow), program acceptance - Q(Iwant), emotional attitude - Q(Ilove), personal status and perseverance $\mathrm{Q}(\mathrm{Iam})$, creative implication - Q(Icreate), concordance with the personal inherited abilities and skills - Q(Icreated), and trust and confidence in the MBT results - Q(Ibelieve). The relative value of the corresponding quality of a specific center defined as $r$ (Center) = $\mathrm{Q}$ (Center)/10, shows the activation level of that center with respect to the proposed or applied MBT program, so the general relative value r(I) mediated over all seven cognitive centers of a patient, fully characterizing the cognitive activation, could be estimated by the following relation:

$$
r(I)=[r(\text { Iknow })+r(\text { Iwant })+r(\text { Ilove })+r(\text { Iam })+r(\text { Icreate })+
$$
$r($ Icreated $)+r($ Ibelieve $)] / 7$

With the numerical values from the diagram shown in Figure 1 , the general relative value of the activation level of the cognitive centers $r(\mathrm{I})=0.7$ is obtained, indicating a positive chance $(r(\mathrm{I})$ $>0.5$ ) of successful MBT application. If multiple diseases or dysfunctions (typically the main and the secondary derivative one) are to be taken into account, an integrate MBT cycle treating the two diseases could be applied, by using two consecutive musictype categories, so two reference $r_{B 1}$ and $r_{B 2}$ diagrams should be prepared, like that described above, each of them elaborated before the MBT application with respect to the addressed specific music program-sequence. Consequently, the general reference relative activation level in a multitask appraisal can be calculated according to the relation: $r=\left(r_{B 1}+w r_{B 2}\right) / 2$, where we would be a "weight" factor of the activation level in the secondary sequence (with values between 0 and 1 , typically 0.5 ), according to the corresponding importance of it in the panoramic scenario of the health status of the patient. We will determine in this way a reference diagram and a reference relative value marked by $r_{R^{*}}$ The improvement in the rehabilitation evolution can be evaluated applying again the NBM type procedure to each cognitive center, and can be observed from the obtained feedback diagram, from the activation level of each cognitive center and from the relative value $\mathrm{r}_{\mathrm{FB}}$. If $\mathrm{r}_{\mathrm{FB}} / \mathrm{r}_{\mathrm{B} 1}>1$, then a favorable evolution of the rehabilitation process is registered; if not, a correction of the music program better adapted to the particular cognitive characteristics of the patient should be applied.

\section{Conclusion}

The rapid increase of the number of the old age adults and the increasing of their lifespan asks an indispensable improvement of some efficient techniques and procedures suitable to be applied to such a category of patients. Moreover, taking into account the high risk of the degenerative neuro-diseases at the old age population and secondary induced dysfunctions, the application of the MBT is a remedial preferential technique for neurorehabilitation on a large spectrum of dysfunctions and disorders, some of them (Alzheimer's and Parkinson's diseases) otherwise incurable. Based 
on the spectacular advances in understanding of consciousness from the informational perspective, it was shown that an efficient MBT could be applied following an adequate procedure, combining the EBM with NBM techniques, allowing to adjust and stimulate the patient participation to the MBT programs by the activation of particular own mental characteristics and engaging them actively in the rehabilitation program. The described procedure fully covers the entire mental cognitive properties of the mind, expressed by the seven cognition centers, each of them with specific distinct functions. The described technique increases therefore the powerful applicative potential of MBT process and its effects, allowing a numerical estimation of the activation level of each center and of an average value of the fully described cognitive characteristics of the patient. An adequate evaluation multitask procedure was also described, characteristic and necessary in old age MBT applicative programs due to the secondary effects (especially anxious and depression induced states) of the main degenerative disease, allowing a suitable evaluation of the MBT performances applied to each patient and their improvement.

\section{Acknowledgment}

This work is dedicated to Adrian Gaiseanu, eminent specialist, son and father and to his family, and to Ana-Maria Gaiseanu, for her high qualities in family, society and profession. The author expresses his grateful thanks to this Journal for kind invitation and professional publication.

In memoriam of the author's loved parents and brother, Distinguished (Magna cum Laude) Professor Emanoil Gaiseanu, Distinguished (Emeritus) Professor Florica Gaiseanu, Professor Constantin Gaiseanu and of the other members of the family.

\section{References}

1. Gaiseanu F (2019) Language patterns and cognitive-sentient reality: Certainty/uncertainty in cognitive-sentient exploration of reality. In: Stephen BS (Ed.), Chapter in Media Models to Foster Collective Human Coherence in the PSYCH ecology. IGI Global, USA, pp. 49-72.

2. Thaut M, Koshimori Y (2020) Chapter 14-neurorehabilitation in aging through neurologic music therapy. Music and the Aging Brain, pp.351382.

3. Gaiseanu F (2020) Informationally assisted equilibrium and health: specific ACC contribution from the perspective of the informational model of consciousness. EC Psychology and Psychiatry 9(5): 37-49.

4. Donatella D, Sarsina R, Elios PD, Patrick J (2010) Music and medicine. Journal of Multidisciplinary Healthcare 3: 137-141.

5. Izet M, Milan M, Belma M (2008) Evidence based medicine new approaches and challenges. Acta Inform Med 16(4): 219-225.

6. Zaharias G (2018) What is narrative-based medicine? (1) Narrativebased medicine. Canadian Family Physician 64(3): 176-180.

7. Mainka S, Spintge R, Thaut M (2016) Music therapy in medical and neurological rehabilitation settings. In: Susan Hallam, Ian Cross, Michael Thaut (Eds.), The Oxford Handbook of Music Psychology. (2 $\left.2^{\text {nd }} e d n\right)$, Germany, pp. 1-22.

8. Galińska E (2015) Music therapy in neurological rehabilitation settings. Psychiatr Pol 49(4): 835-846.
9. Koelsch S, Jacobs A, Liebal K, Delius G, Scheve C, et al. (2015) The quartet theory of human emotions: An integrative and neurofunctional model. Physics of Life Reviews 13: 1-27.

10. Koelsch S, Skeie G (2020) Chapter 1-the musical brain. Music and the Aging Brain pp. 1-40.

11. Sescousse G, Caldu X, Segura B, Dreher JC (2013) Processing of primary and secondary rewards: A quantitative meta-analysis and review of human functional neuroimaging studies. Neuroscience \& Biobehavioral Reviews 37(4): 681-696.

12. Linnemann A, Kappert MB, Fischer S, Doerr JM, Strahler J, et al. (2015) The effects of music listening on pain and stress in the daily life of patients with fibromyalgia syndrome. Front Hum Neurosci 9: 434.

13. Dalla BS (2015) The oxford handbook of music psychology. In: Susan Hallam, Ian Cross, and Michael Thaut (Eds.), ( $2^{\text {nd }}$ edn), University of Cambridge, England, pp. 1-17.

14. Gaiseanu F (2019) Destiny or free will decision? A Life Overview from the Perspective of an Informational Modeling of Consciousness Part I: Information, Consciousness and Life Cycle. Gerontology \& Geriatric Studies 4(3): 400-405.

15. Gaiseanu F (2018) Destiny or free will decision? a life overview from the perspective of an informational modeling of consciousness part II: attitude and decision criteria, free will and destiny. Gerontology \& Geriatric Studies 4(1): 354-360.

16. Gaiseanu F (2019) The informational model of consciousness: mechanisms of the embodiment/disembodiment of information. Neuro Quantology 17(4): 1-17.

17. Gaiseanu F (2019) Informational Neuro-Connections of the Brain with the Body Supporting the Informational Model of Consciousness. Archives in Neurology \& Neuroscience 4(1): 1-6.

18. Gaiseanu F (2020) Information-matter bipolarity of the human organism and its fundamental circuits: from philosophy to physics/neurosciencesbased modeling. Philosophy Study 10(2): 107-118.

19. Gaiseanu F (2017) Quantum-assisted process of disembody under near-death conditions: an informational-field support model. Neuro Quantology 15(1): 4-9.

20. Gaiseanu F (2017) An information-based model of consciousness fully explaining the mind normal/paranormal properties. Neuro Quantology 15(2): 132-140.

21. Gaiseanu F (2020) Info-relational cognitive operability of the posterior cingulate cortex according to the informational model of consciousness. International Journal of Psychological and Brain Sciences 5(4): 61-68.

22. Gaiseanu F (2019) Informational model of consciousness: from philosophic concepts to an information science of consciousness. Philosophy Study 9(4): 181-196.

23. Gaiseanu F (2019) The silent voice of those who are no longer: transgenerational transmission of information from the perspective of the informational model of consciousness. Gerontology \& Geriatric Studies 5(1): 482-488.

24. Gaiseanu F (2019) Epigenetic information-body interaction and information-assisted evolution from the perspective of the informational model of consciousness. Archives in Biomedical Engineering \& Biotechnology 2(2): 1-6.

25. Chen MC, Tsai PL, Huang YT, Lin KC (2013) Pleasant music improves visual attention in patients with unilateral neglect after stroke. Brain Inj 27(1): 75-82.

For possible submissions Click below: 